\title{
An Immunoprotective Molecule, the Major Secretory Protein of Legionella pneumophila, Is Not a Virulence Factor in a Guinea Pig Model of Legionnaires' Disease
}

\author{
Steven J. Blander, ${ }^{*}$ Lisa Szeto, ${ }^{\ddagger}$ Howard A. Shuman, ${ }^{\ddagger}$ and Marcus A. Horwitz* \\ *Division of Infectious Diseases, Department of Medicine, University of California at Los Angeles School of Medicine, \\ Center for the Health Sciences, Los Angeles, California 90024; ${ }^{\ddagger}$ Department of Microbiology, \\ College of Physicians and Surgeons of Columbia University, New York 10032
}

\begin{abstract}
We have examined whether a molecule that is capable of inducing immune protection, the major secretory protein (MSP) of Legionella pneumophila, is required for virulence in a guinea pig model of Legionnaires' disease. To do so, we have compared the virulence in guinea pigs of an isogenic pair of $L$. pneumophila, Philadelphia 1 strain, one of which produces MSP (MSP+) and one of which does not (MSP-).

Both the MSP- strain and the MSP+ strain of $L$. pneumophila are highly virulent for guinea pigs, inducing similar signs and progression of illness. Both strains are lethal and have comparable $\mathrm{LD}_{50} \mathrm{~S}$ and $\mathrm{LD}_{100}$. Both strains multiply in the lungs of guinea pigs at a similar rate, and both strains produce indistinguishable pathological lesions in the lungs. Both strains maintain a stable phenotype with guinea pig passage, i.e., the MSP- strain does not regain the capacity to secrete MSP and the MSP+ strain retains its capacity to secrete MSP after lung passage.

Although vaccination with MSP induces strong protective immunity in the guinea pig against lethal aerosol challenge with $L$. pneumophila, this protective immunogen is not required in its intact proteolytically active form for the expression of virulence by the intracellular pathogen $L$. pneumophila. This demonstrates that a protective immune response need not necessarily be directed against a virulence determinant and suggests that any molecule that allows the host immune system to detect and act against an intracellularly sequestered pathogen may potentially serve as a protective immunogen against such a pathogen. (J. Clin. Invest. 1990: 86:817-824.) Key words: intracellular parasitism • protective immunity • protease - isogenic mutant • vaccine
\end{abstract}

\section{Introduction}

Legionella pneumophila is a facultative intracellular bacterial pathogen that causes $80-85 \%$ of the cases of Legionnaires'

Address reprint requests to Dr. Horwitz, Division of Infectious Diseases, Department of Medicine; UCLA School of Medicine, 10833 Le Conte Avenue, Los Angeles, CA 90024.

Received for publication 11 January 1990 and in revised form 25 April 1990.

J. Clin. Invest.

(c) The American Society for Clinical Investigation, Inc. 0021-9738/90/09/0817/08 $\$ 2.00$

Volume 86, September 1990, 817-824 disease, a serious and often fatal form of pneumonia $(1,2)$. Several potential virulence factors have been identified in $L$. pneumophila including an acid phosphatase $(3,4)$, phospholipase $(5)$, the mip gene product $(6,7)$, and proteases $(8-12)$. Except for the mip gene product, which potentiates infection with $L$. pneumophila, none of these factors has been shown to be necessary for the full expression of virulence by $L$. pneumophila.

A previous study from one of our laboratories demonstrated that the L. pneumophila Philadelphia 1 major secretory protein (MSP), ${ }^{1}$ a metalloprotease of apparent mol wt 39,000, induces strong cell-mediated and protective immunity in a guinea pig model of Legionnaires' disease. This study showed that $(a)$ guinea pigs sublethally infected with aerosolized $L$. pneumophila develop a strong cell-mediated immune response to MSP, as measured by splenic lymphocyte proliferation and cutaneous delayed-type hypersensitivity; (b) guinea pigs vaccinated subcutaneously with purified MSP develop strong humoral and cell-mediated immune responses to MSP; and (c) guinea pigs vaccinated with MSP develop a very high level of protective immunity against lethal aerosol challenge with $L$. pneumophila (13).

Other investigators have suggested a pathogenic role for a major protease of $L$. pneumophila (10-11). Conlan et al. inoculated guinea pigs intranasally or intratracheally with an $L$. pneumophila protease of nearly the same apparent molecular weight as MSP and observed inflammatory pathologic lesions which appeared similar to those produced by aerosolization of $L$. pneumophila into the lungs of guinea pigs $(10,11)$. Keen and Hoffman (14) and Quinn and Tompkins (15) have demonstrated that MSP has cytotoxic activity.

Traditionally, vaccinogens have been viewed as inducing protective immunity to infectious agents by stimulating an immune response that is capable of neutralizing virulence determinants such as toxins, adhesins, resistance factors, etc. Thus, if MSP were a virulence determinant, one way that vaccination with MSP might induce protective immunity against L. pneumophila would be by neutralizing MSP activity. Alternatively, however, in the case of an intracellular pathogen, vaccinogens might potentially induce protective immunity by expanding a population of effector cells of the immune system that are capable of recognizing and exerting an antimicrobial

1. Abbreviations used in this paper: CYEA, charcoal yeast extract agar; EYB, egg yolk buffer, $m s p A$, structural gene for MSP; MSP+, MSPpositive strain of $L$. pneumophila; MSP, major secretory protein; MSP-, MSP-negative strain of $L$. pneumophila; YEB, yeast extract broth. 
effect against infected host cells. Thus, another way that vaccination with MSP might induce protective immunity against $L$. pneumophila is by targetting immune cells to mononuclear phagocytes infected with this pathogen.

Recently, one of our laboratories has constructed a mutant of L. pneumophila, Philadelphia 1, which does not produce any detectable intact MSP (15a). This has allowed us to investigate whether MSP is required for virulence of $L$. pneumophila in the animal host. In this study, we have compared the virulence of the MSP-negative strain of $L$. pneumophila (MSP-) with that of its parental MSP-positive strain (MSP+) in the guinea pig model of Legionnaires' disease. We shall show that both the MSP- and MSP+ strain of L. pneumophila (a) are virulent for guinea pigs; $(b)$ produce a pneumonic illness and death at comparable aerosolized doses; $(c)$ multiply in the lungs of guinea pigs at comparable rates; and $(d)$ produce similar pathological changes.

This study demonstrates that, although MSP is a potent immunogen with the capacity to induce protective immunity, the intact proteolytically active molecule is not required for virulence in the guinea pig model of Legionnaires' disease. Therefore, this study demonstrates that vaccinogens can induce protective immunity in ways other than by neutralizing virulence determinants, such as by enhancing the capacity of the immune system to detect and respond effectively to an intracellular pathogen.

\section{Methods}

Animals. Male Hartley strain guinea pigs weighing $450-500 \mathrm{~g}$, purchased from Charles River Breeding Laboratories (North Wilmington, MA) were housed, no more than four to a stainless steel cage, and allowed free access to ascorbic acid-fortified chow and water. Guinea pigs were observed in the vivarium for signs of illness for $1 \mathrm{wk}$ before challenge with $L$. pneumophila to ensure that they were healthy; no illnesses or deaths occurred in guinea pigs before challenge. After challenge, guinea pigs were held in filter-top cages for 1 wk. During this time, they were observed for signs of illness and survival was quantitated.

Media. Each liter of yeast extract broth (YEB) was prepared with $10 \mathrm{~g}$ of ACES (Sigma Chemical Co., St. Louis, MO), $10 \mathrm{~g}$ yeast extract (Difco Laboratories, Detroit, MI), $0.4 \mathrm{~g}$ L-cysteine HCL (Fisher Scientific Co., Fair Lawn, NJ), and $0.25 \mathrm{~g}$ ferric pyrophosphate (Sigma Chemical Co.), adjusted to a pH of 6.9 with $10 \mathrm{~N} \mathrm{KOH}$, and filter sterilized. Egg yolk buffer (EYB) with or without 1\% BSA (Miles Laboratories, Inc., Naperville, IL) was prepared as previously described (1).

Agar. Modified charcoal yeast extract agar (CYEA) was prepared as described (1). Modified CYEA without cysteine and tryptic soy agar with $5 \%$ sheep blood were used to assay for contamination of non- $L e$ gionella species as described (1).

Bacteria. An isogenic pair of Legionella pneumophila, Philadelphia 1 strain, one of which is MSP+ (LS2029) and one of which is MSP(LS2102) was constructed (15a). The MSP- mutant was constructed by insertion of transposon $\mathrm{Tn} 9$ in the structural gene for MSP, $m s p A$. The $m s p A$ gene was identified in a library of $L$. pneumophila, Philadelphia 1 strain, genomic DNA after transfer to an Escherichia coli host strain. Transposon $\operatorname{Tn} 9$ insertions in the $m s p A$ gene were constructed in $E$. coli. A total of six independent $\operatorname{Tn} 9$ insertions throughout the MSP coding sequence were transferred to the genome of the $L$. pneumophila, Philadelphia 1 strain derivative, LS2029. All six mutant strains exhibited the same low level of caseinolytic activity (0.2\%) compared to LS2029. In addition, all six strains retained their viru- lence for human macrophages differentiated from the HL-60 cell line. One mutant strain, LS2102, was studied in detail and was found to grow as well as the parental LS2029 strain within HL-60-derived macrophages. LS2 102 was found to produce a small amount of a polypeptide of lower apparent molecular weight than MSP, which appeared to cross-react with polyclonal rabbit anti-MSP antiserum; the amount of this polypeptide produced by the mutant was $\sim 1 \%$ of the amount of MSP produced by the wild type. This polypeptide may represent an amino terminal fragment of MSP (15a).

The MSP+ and MSP- bacteria selected for study were grown on CYEA; washed with EYB; flash frozen in aliquots of $10^{11} \mathrm{CFU} / \mathrm{ml}$; stored at $-70^{\circ} \mathrm{C}$; and tested for the presence of contaminating bacteria as previously described (1).

Infection of guinea pigs. Guinea pigs were infected by exposure to aerosols of bacteria in the same lucite aerosol chamber described in detail in a previous study (16). Briefly, the chamber had sealable portals for introducing or removing guinea pigs, an aerosol inlet at the chamber ceiling, and an aerosol nebulizer system (Dart Industries, Ocala, FL). A vacuum pump (Gast Mfg. Co., Benton Harbor, MI) delivered positive pressure to the nebulizer and negative pressure to the chamber. Guinea pigs were infected by exposure for $30 \mathrm{~min}$ to an aerosol generated from a $10-\mathrm{ml}$ suspension containing $1.5 \times 10^{5}$ to 1.0 $\times 10^{9} \mathrm{CFU} / \mathrm{ml}$ of the MSP- strain of $L$. pneumophila, or $2.5 \times 10^{7}$ to $1.0 \times 10^{9} \mathrm{CFU} / \mathrm{ml}$ of the MSP+ strain of L. pneumophila. After the pump to the nebulizer was shut off, the guinea pigs were held inside the chamber for an additional $10 \mathrm{~min}$ before being removed and placed in filter-top cages, three to a cage. Guinea pigs shared cages only with animals exposed to the same type and dose of aerosol. After the challenge, the number of survivors in each group of animals was quantitated over a 7-d period. Each experiment was performed in duplicate.

Quantitation of the MSP- and MSP+ strain of L. pneumophila in pulmonary tissue. In two independent experiments, guinea pigs were infected with an aerosol generated from a 10-ml suspension of 2.5 $\times 10^{8} \mathrm{CFU} / \mathrm{ml}$ of either the MSP- or MSP+ strain of L. pneumophila. In a third experiment, guinea pigs were infected with an aerosol generated from a $10-\mathrm{ml}$ suspension of $7.5 \times 10^{7} \mathrm{CFU} / \mathrm{ml}$ for the MSP- and $1.5 \times 10^{8} \mathrm{CFU} / \mathrm{ml}$ for the MSP+ strain of $L$. pneumophila. At various time points 3 to $72 \mathrm{~h}$ after challenge, one guinea pig infected with an MSP- and one infected with an MSP+ strain of $L$. pneumophila were killed by hypercarbia. The animal was soaked first in $7 \times$ cleaning solution (Linbro Chemical Co., New Haven, CT) and then in $70 \%$ ethanol to decontaminate the skin, and the lungs were removed by sterile technique. The left lung was set aside for pathological examination (see below). The right lung was placed in $10 \mathrm{ml}$ of sterile EYB, ground thoroughly with a mortar and pestle, and serially diluted in EYB with $1 \%$ BSA. CFU was determined by plating serial logarithmic dilutions of the lung suspension in EYB with 1\% BSA in triplicate on CYEA. On serial plates, CFU decreased by $\sim 10$-fold from plate to plate, i.e., by the dilution factor, as expected. Lung suspensions were not subjected to hypotonic lysis before plating because control studies demonstrated that few intact lung cells remain in the suspensions after grinding with the mortar and pestle and that hypotonic lysis does not alter bacterial counts. The lung suspensions were also tested for the presence of contaminating bacteria as described (1), and none were contaminated.

Pathology. The left lung from a guinea pig infected with either the MSP - or the MSP+ strain of L. pneumophila was excised 48 and $72 \mathrm{~h}$ after infection as described in the preceding paragraph. Tissue samples from three areas of each lung were fixed in $10 \%$ formalin, embedded in paraffin, sliced with a microtome, stained with hematoxylin and eosin, and examined by light microscopy. The experiment was performed three times.

Secretion of MSP by the MSP- and MSP+ strains of L. pneumophila before and after passage through guinea pig lungs. Extracellular proteins were obtained from the MSP- and MSP+ strain of $L$. pneumophila before and after passage in guinea pigs. Passaged strains of $L$. pneumophila were cultured from guinea pig lungs $72 \mathrm{~h}$ after infection. 
Table I. Survival Rates of Guinea Pigs Challenged with Aerosols Containing Various Concentrations of the MSP- or MSP+ strain of L. pneumophila

Number of guinea pigs surviving per number challenged

\begin{tabular}{|c|c|c|c|c|c|c|}
\hline \multirow{2}{*}{$\begin{array}{l}\text { Concentration of bacteria } \\
\text { in suspension from } \\
\text { which aerosol generated }\end{array}$} & \multicolumn{3}{|c|}{ MSP- L. pneumophila } & \multicolumn{3}{|c|}{ MSP+ L. pneumophila } \\
\hline & Exp. A & Exp. B & Total (\%) & Exp. A & Exp. B & Total (\%) \\
\hline$C F U / m l$ & & & & & & \\
\hline $1.5 \times 10^{5}$ & $3 / 3$ & - & $3 / 3(100)$ & - & - & - \\
\hline $1.5 \times 10^{6}$ & $3 / 3$ & - & $3 / 3(100)$ & - & - & - \\
\hline $2.5 \times 10^{7}$ & $2 / 4$ & - & $2 / 4(50)$ & $4 / 4$ & - & $4 / 4(100)$ \\
\hline $5.0 \times 10^{7}$ & $2 / 4$ & $3 / 3$ & $5 / 7(71)$ & $2 / 4$ & $1 / 3$ & $3 / 7(43)$ \\
\hline $1.0 \times 10^{8}$ & $1 / 4$ & $0 / 4$ & $1 / 8(13)$ & $0 / 4$ & $0 / 4$ & $0 / 8(0)$ \\
\hline $2.5 \times 10^{8}$ & - & $1 / 4$ & $1 / 4(25)$ & $2 / 4$ & $0 / 4$ & $2 / 8(25)$ \\
\hline $5.0 \times 10^{8}$ & $0 / 2$ & $0 / 4$ & $0 / 6(0)$ & - & $0 / 4$ & $0 / 4(0)$ \\
\hline $1.0 \times 10^{9}$ & - & $0 / 4$ & $0 / 4(0)$ & $0 / 3$ & $0 / 4$ & $0 / 7(0)$ \\
\hline
\end{tabular}

In two independent experiments (A and B), groups of guinea pigs were challenged with an aerosol generated from a suspension containing various concentrations of the MSP- or MSP+ strain of L. pneumophila, as indicated, and survival was quantitated.

The extracellular proteins were obtained as previously described with minor modifications (13). Briefly, the bacteria were grown on CYEA plates; suspended at an initial OD of 0.05 at $540 \mathrm{~nm}$ in $10 \mathrm{ml}$ of prewarmed YEB; and grown in $55 \mathrm{ml}$ screw-cap conical polypropylene tubes for $20 \mathrm{~h}$ at $37^{\circ} \mathrm{C}$ on a platform rotating at $120 \mathrm{rpm}$. When a final OD of 1.0 was reached, the culture was tested for the presence of contaminants by inoculating CYEA without L-cysteine and tryptic soy agar with $5 \%$ sheep blood. The bacteria were pelleted by centrifugation, and the supernate was decanted and filtered to remove residual bacteria. EDTA was added to each filtrate to a concentration of $0.01 \mathrm{M}$.

The extracellular proteins thus obtained were examined by SDSPAGE (17) and Western immunoblot analysis (18) using the alkaline phosphatase technique (19) with the following modifications. For both blocking and the dilution of antibody reagents, a solution containing $0.01 \mathrm{M}$ Tris, $0.15 \mathrm{M} \mathrm{NaCl}, 0.5 \%$ Tween- 20 , and $0.02 \%$ sodium azide was used. Affinity purified monospecific anti-MSP rabbit antibody $(1.5 \mathrm{ng} / \mathrm{ml})$ was used as a probe for MSP; a 1:1,000 dilution of alkaline phosphatase-conjugated goat anti-rabbit IgG (heavy and light chain specific) (Cappel Laboratories, West Chester, PA) was used as the enzyme conjugate; and an enzyme substrate solution prepared by adding $500 \mu \mathrm{l}$ of $0.1 \%$ nitroblue tetrazolium (Sigma Chemical Co.) in distilled water and $100 \mu \mathrm{l}$ of $0.5 \% 5$-bromo-4-chloro-indolyl phosphate (Sigma Chemical Co.) in anhydrous dimethyl formamide (Pierce Chemical Co., Rockford, IL) to $9.5 \mathrm{ml}$ of $0.05 \mathrm{M}$ Tris, pH 10, containing $0.03 \mathrm{M} \mathrm{MgCl}_{2}$, was used to develop the reaction. The reaction was halted by rinsing the immunoblot with distilled water.

Proteolytic activity of extracellular proteins produced by the MSPand MSP+ strains before and after passage through guinea pig lungs. The extracellular proteins produced by the MSP - and MSP+ strain of L. pneumophila before and after passage through guinea pig lungs were analyzed for proteolytic activity by a colorimetric assay. A 50-mg substrate of hide powder azure was dissolved in $2 \mathrm{ml}$ of a diluting buffer consisting of $0.1 \mathrm{M}$ sodium phosphate and $8.5 \%$ sodium chloride, $\mathrm{pH}$ 7.4. The extracellular proteins of the bacteria, obtained as described in the previous section, or YEB (control) were added in $200-\mu l$ aliquots to the hide powder azure mix. The mixture was rotated at $150 \mathrm{rpm}$ at $37^{\circ} \mathrm{C}$ for $30 \mathrm{~min}$ and chilled to $4^{\circ} \mathrm{C}$. The supernates were then aspirated, and the $O D$ read at $595 \mathrm{~nm}$ on a spectrophotometer (Junior model 35; Perkin-Elmer Corp., Oak Brook, IL) with the OD of the control set to zero.

Statistics. Guinea pig survival data were analyzed using the logistic model: $p=1 /[1+\exp (x-u /$ sigma $)]$ where $p=$ proportion dead at log dose $x ; x=$ the dose; $u=\mathrm{LD}_{50} ;$ and sigma $=$ logit slope parameter (20).
Calculations of $\mathrm{LD}_{50}, \mathrm{SE}, P$ value, sigma, and power of the test were obtained with the Proc Catmod module of the SAS computer program (SAS Institute, Cary, NC).

\section{Results}

The MSP-strain of L. pneumophila is virulent for guinea pigs at challenge doses comparable to those of the MSP+ strain. In two independent experiments, we infected groups of guinea pigs with various aerosolized doses of the MSP- and MSP+ strain of $L$. pneumophila and quantitated survival. Both strains were lethal for guinea pigs (Table I). For both strains, $100 \%$ of guinea pigs were killed by aerosols generated from a suspension containing $5 \times 10^{8}$ or more CFU of $L$. pneumophila $/ \mathrm{ml}$. The $50 \%$ lethal dose $\left(\mathrm{LD}_{50}\right)$ for the MSP-strain was an aerosol generated from a suspension containing $4.8 \times 10^{7}$ $\mathrm{CFU} / \mathrm{ml}\left(\log \mathrm{LD}_{50} \pm \mathrm{SE}=7.68 \pm 2.19\right)$. Similarly, the $\mathrm{LD}_{50}$ for the MSP+ strain was an aerosol generated from a suspension containing $4.9 \times 10^{7} \mathrm{CFU} / \mathrm{ml}\left(\log \mathrm{LD}_{50} \pm \mathrm{SE}=7.69 \pm 2.20\right)$. The difference in $\mathrm{LD}_{50} \mathrm{~S}$ was not significant $(P=0.73)$. The $\mathrm{SE}$ for the difference $(0.01)$ between the $\mathrm{LD}_{50}$ 's for the two strains was $0.10(\log \mathrm{CFU} / \mathrm{ml})$. Given this SE of the difference, at the $5 \%$ alpha level, the power for detecting a difference of $0.5 \mathrm{log}$ $\mathrm{CFU} / \mathrm{ml}$ or larger between the $\mathrm{LD}_{50}$ 's of the two strains was over $90 \%$. The shape of the guinea pig survival curve (proportion surviving by log dose) for the two strains was also similar; sigma \pm SE for the MSP- strain was $0.29 \pm 0.08(\log \mathrm{CFU} / \mathrm{ml})$, while sigma $\pm \mathrm{SE}$ for the MSP+ strain was $0.27 \pm 0.07$, a difference which was not significant $(P=0.72)$.

Guinea pigs infected with the MSP- and MSP+ strain of L. pneumophila exhibited similar onset and signs of illness including respiratory distress, decreased activity, and decreased feeding.

The MSP - and MSP+ strain of L. pneumophila multiply at the same rate in guinea pig lungs. To assess the capacity of the MSP- and MSP+ strain of $L$. pneumophila to multiply in the lungs of guinea pigs, we challenged guinea pigs with a lethal 


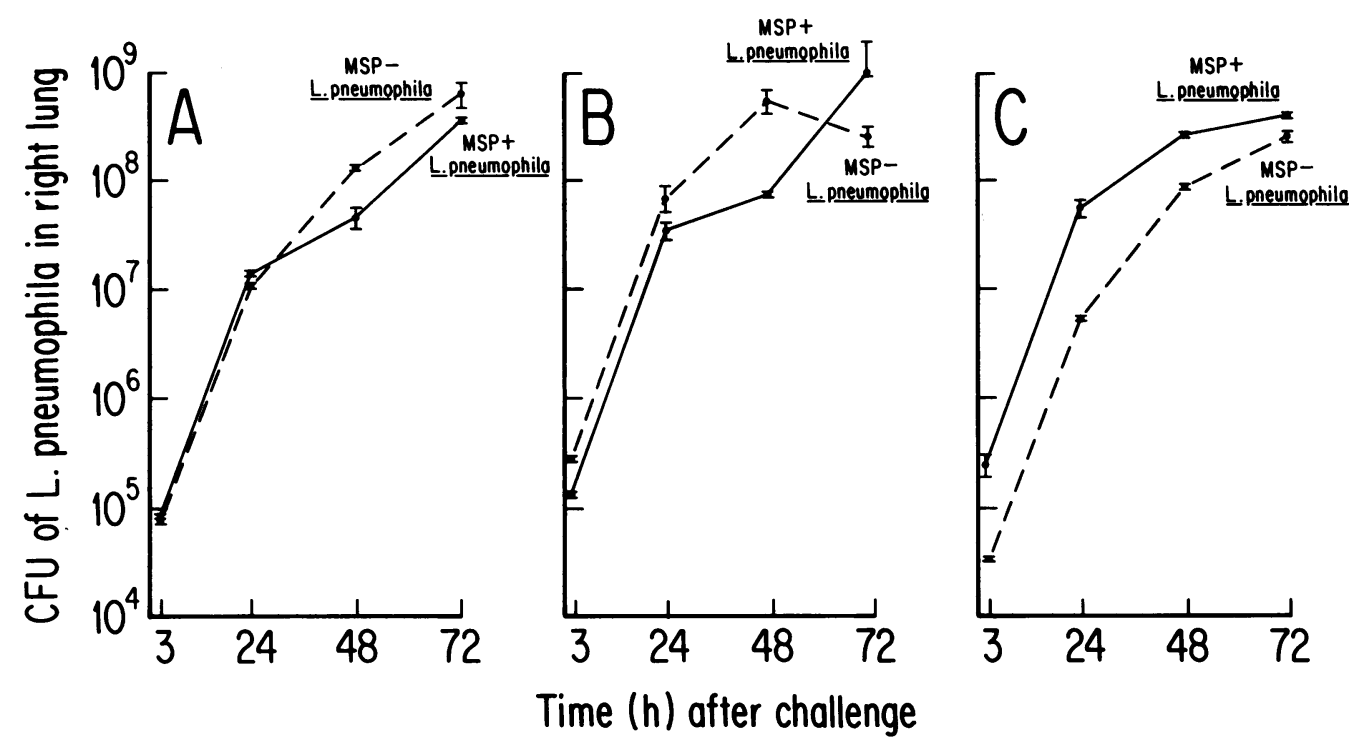

Figure 1. The MSP- and MSP+ strain of L. pneumophila multiply in the guinea pig lung at comparable rates. In three independent experiments $(A-C)$, guinea pigs were challenged with an aerosol containing the MSPor MSP+ strain of L. pneumophila. In experiments A and $B$, guinea pigs were exposed to an aerosol generated from a suspension containing $2.5 \times 10^{8} \mathrm{CFU} / \mathrm{ml}$ of either strain. In experiment $\mathrm{C}$, guinea pigs were exposed to an aerosol generated from a suspension containing 7.5 $\times 10^{7} \mathrm{CFU} / \mathrm{ml}$ of the MSPstrain and $1.5 \times 10^{8} \mathrm{CFU} / \mathrm{ml}$ of the MSP+ strain of $L$. pneumophila. At 3, 24, 48,

or $72 \mathrm{~h}$ after challenge, as indicated, one guinea pig infected with an MSP- strain and one guinea pig infected with an MSP+ strain was killed and CFU of $L$. pneumophila in the right lung was determined as described in the text. Each point represents the mean \pm SEM of triplicate measurements for each lung.

dose of each of the strains, killed animals $3,24,48$, or $72 \mathrm{~h}$ later, and assayed CFU of L. pneumophila in the right lung. We performed three independent experiments (Fig. 1).

In all three experiments, both the MSP- and MSP+ strain multiplied approximately four logs over the course of the experiments. In the first two experiments (Fig. 1, $A$ and $B$ ), where the guinea pigs were infected with approximately the same number of MSP - and MSP+ L. pneumophila, the two strains of bacteria multiplied at essentially the same rate, although there were inconsistent differences at one time point or another. In the third experiment (Fig. $1 C$ ), where guinea pigs infected with the MSP - strain received $\sim 1$ log fewer bacteria than guinea pigs infected with the MSP+ strain, the bacteria also multiplied at comparable rates so that the growth curves of the two strains parallelled each other throughout the course of the experiment.

$M S P$ - and MSP+ strains of L. pneumophila cause similar pathological lesions in the lungs of infected guinea pigs. To compare the pathological lesions induced by the MSP- and MSP+ strain of L. pneumophila in the lungs of guinea pigs, we infected guinea pigs with the bacteria, excised the left lung 48 and $72 \mathrm{~h}$ later, processed the tissues for pathological examination, and examined them by light microscopy. In lung specimens from guinea pigs infected with either strain of $L$. pneumophila, we observed infiltrates of mononuclear and polymorphonuclear cells, pink exudates in some alveoli, evidence of hemorrhage, and consolidation of alveolar airspaces (Fig. 2). Inflammatory cell infiltration and consolidation increased with time. Some areas of nodular mononuclear cell infiltration were seen. There was no difference in the pathology induced by the MSP- and MSP+ strain of L. pneumophila.

The MSP- and MSP+ strain do not change phenotype with lung passage. To examine the possibility that the MSPstrain of L. pneumophila mutates and regains the capacity to produce MSP during the course of infection, we examined the extracellular proteins of the MSP- and MSP+ strains before and after infection of guinea pigs. The extracellular proteins were examined for the presence of MSP by SDS-PAGE and Western immunoblot analysis. The MSP- strain of L. pneumophila did not produce MSP before or $72 \mathrm{~h}$ after passage in guinea pig lung. In contrast, the MSP+ strain of L. pneumophila produced MSP both before and $72 \mathrm{~h}$ after passage in guinea pig lung (Fig. 3). Thus, with regard to MSP production, the MSP- and MSP+ strain do not change phenotype after lung passage.

The proteolytic activity of the MSP- and MSP+ strain does not change with lung passage. To determine if the proteolytic activity of the extracellular proteins of the MSP- and MSP+ strain of $L$. pneumophila remains stable with guinea pig passage, we assayed proteolytic activity of the strains before and after guinea pig passage (Table II). The extracellular proteins of the MSP+ strain exhibited abundant proteolytic activity both before and after passage through guinea pig lung; there was no significant difference in proteolytic activity before and after passage ( $P>0.05$ by the $t$ test, two-tailed). In contrast, the extracellular proteins of the MSP- strain exhibited little proteolytic activity either before or after passage through guinea pig lung. The OD readings for the MSP-strain both before and after passage were $<0.05$, below the range for accurate spectrophotometric readings. Thus, also with regard to proteolytic activity, the MSP- and MSP+ strain maintain their phenotype with lung passage.

\section{Discussion}

This study demonstrates that MSP production by L. pneumophila is not required for the full expression of virulence by this intracellular bacterial pathogen in the guinea pig model of 

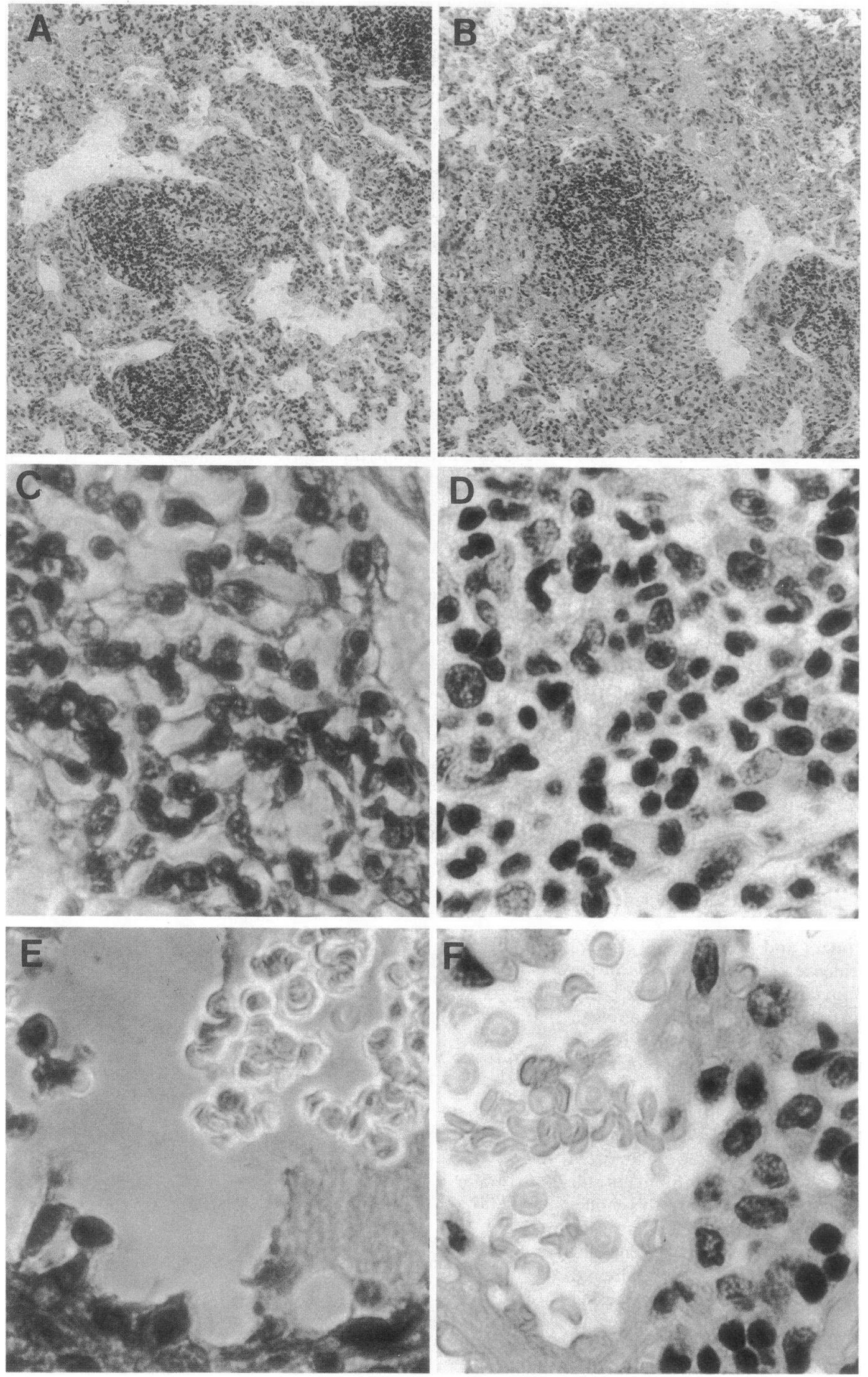

Figure 2. Lungs of guinea pigs infected with MSPand MSP+ L. pneumophila exhibit identical histopathological features. Guinea pigs were infected with an aerosolized dose of either MSP- L. pneumophila $(A, C, E)$ or $\mathrm{MSP}+L$. pneumophila $(B, D, F) .(A$ and $B)$ $(\times 100)$ Lungs of guinea pigs infected with either the MSP- or MSP+ strain contain inflammatory cell infiltrates with areas of nodularity, alveolar exudates, and consolidation. ( $C$ and $D$ ) $(\times 1000)$ The nodular areas of inflammatory cells observed in A and B, respectively, are composed primarily of mononuclear cells. ( $E$ and $F$ ) $(\times 1000)$ Intraalveolar hemorrhage and interalveolar mononuclear cells in the lungs of guinea pigs infected with either of the two strains. $(E)$ An intraalveolar exudate. (Hematoxylin and eosin stain.) 


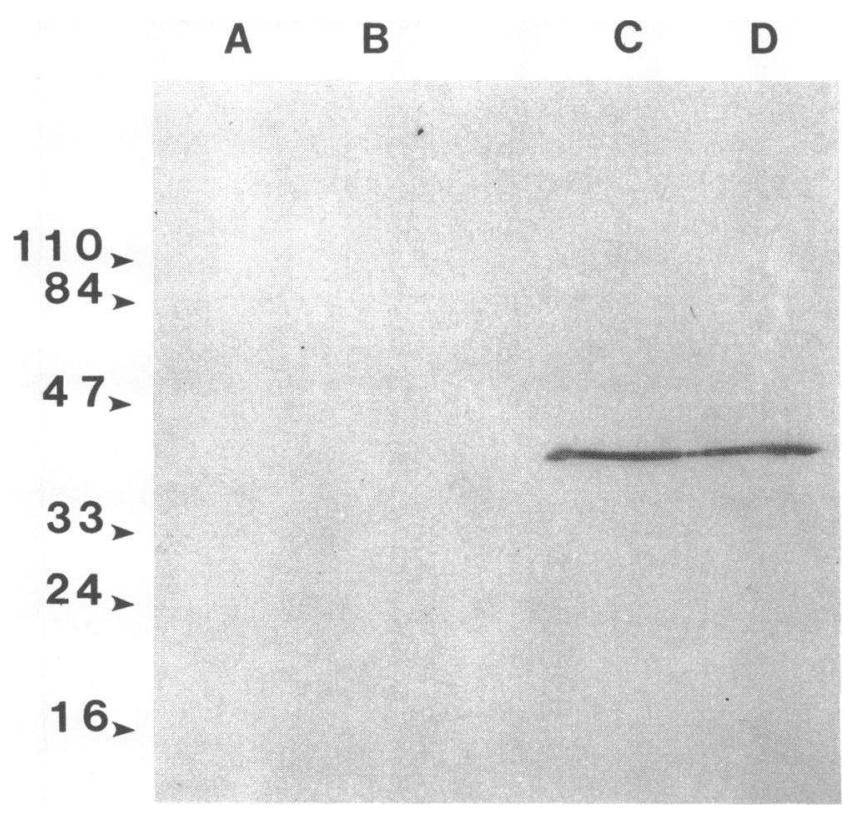

Figure 3. The MSP- and MSP+ strain do not change phenotype with lung passage. Extracellular proteins were obtained from the MSP- and MSP+ strain of $L$. pneumophila before and $72 \mathrm{~h}$ after passage in guinea pigs as described in the text. The proteins were subjected to SDS-PAGE, transferred to nitrocellulose, and probed with an affinity-purified monospecific anti-MSP antibody. Molecular weight markers are shown in kilodaltons. Lane $A, \mathrm{MSP}-$ strain before lung passage; lane $B, \mathrm{MSP}-$ strain after lung passage; lane $C$, MSP+ strain before lung passage; and lane $D, M S P+$ strain after lung passage.

Legionnaires' disease. Both the MSP - and MSP+ strain were highly virulent for guinea pigs. The two strains induced similar signs and progression of illness. The two strains were lethal at comparable doses and had similar $\mathrm{LD}_{50}$ 's. The two strains multiplied in the lungs of guinea pigs at comparable rates. The two strains produced indistinguishable pathological lesions in the guinea pig lung.

This study shows that intact and proteolytically active MSP is not required for virulence of $L$. pneumophila in the guinea pig model, but does not completely rule out the possibility that an amino terminal fragment of MSP plays a role in virulence. The nature of the $\operatorname{Tn} 9$ insertion mutation is such that an amino terminal fragment of MSP lacking proteolytic activity is likely produced. If MSP had an additional activity independent of its proteolytic activity, if this activity were specified by the amino terminal fragment of MSP, and if the residual amount of this fragment produced by the mutant were sufficient for the expression of virulence, then MSP might still play a role in the virulence of $L$. pneumophila in this model system. This seems unlikely because all $6 m s p A$ mutants with Tn 9 insertions throughout the gene retain their virulence for human macrophages. In any case, this issue could be addressed directly in future studies by sequencing the $m s p A$ gene, using this information to construct a deletion of the $m s p A$ gene that does not interfere with the production of other proteins encoded by genes adjacent to the $m s p A$ gene, and studying the virulence potential of the resultant mutant L. pneumophila in the guinea pig model.

The phenotype of the MSP - and MSP+ strain remains stable through passage in guinea pig lung. The MSP- strain did not regain the capacity to secrete MSP after lung passage and the MSP+ strain retained its capacity to secrete MSP after lung passage. Parallelling this, the MSP- strain remained deficient in proteolytic activity through passage in guinea pig lung, while the MSP+ strain retained its high level of proteolytic activity through lung passage. This demonstrates that the virulence of the MSP - strain is not due to a reversion to MSP positivity in vivo, and confirms that MSP is not required for virulence.

Consistent with these observations, in unpublished studies, Blander and Horwitz have identified a naturally occurring (nongenetically manipulated) strain of $L$. pneumophila and a naturally occurring non-pneumophila species of Legionella that do not secrete MSP but are nevertheless still virulent for guinea pigs. The aerosolized doses of these strains that are $100 \%$ lethal are comparable to those of the MSP- and MSP+ strains described in this paper.

In a previous study, Conlan et al. inoculated guinea pigs intranasally or intratracheally with a protease of approximately the same apparent molecular weight $(41,000$ and $42,000)$ as MSP $(39,000)$ and observed pathological lesions in the lungs that were similar in appearance to those observed after aerosolization of $L$. pneumophila into guinea pigs (10, 11). Our finding that the MSP-strain produces lesions in the lung that are indistinguishable from those produced by the MSP+ strain demonstrates that the characteristic pathological lesions in the guinea pig lung infected with $L$. pneumophila are not caused by MSP.

The finding in a previous study (13) from one of our laboratories that guinea pigs sublethally infected with $L$. pneumophila develop a strong cell-mediated immune response to MSP indicates that MSP is produced during infection. However, there is no evidence that MSP produced by L. pneumophila multiplying intracellularly in lung macrophages is released

Table II. Proteolytic Activity of the MSP- and MSP+ Strain of L. pneumophila before and after Lung Passage

\begin{tabular}{llccc}
\hline $\begin{array}{c}\text { Strain of } \\
\text { L. pneumophila }\end{array}$ & $\begin{array}{c}\text { Time } \\
\text { relative } \\
\text { to passage }\end{array}$ & $n^{*}$ & OD $(595 \mathrm{~nm})$ & $\begin{array}{c}\text { \% Proteolytic activity } \\
\text { compared to MSP+ } \\
\text { strain before passage }\end{array}$ \\
\hline & & & Mean $\pm S E$ & \\
MSP+ & Before & 4 & $0.82 \pm 0.09$ & - \\
MSP+ & After & 3 & $0.58 \pm 0.05$ & 70.1 \\
MSP- & Before & 5 & $-0.0002 \pm 0.002$ & 0.0 \\
MSP- & After & 4 & $0.016 \pm 0.006$ & 1.9 \\
& & & & \\
\hline
\end{tabular}

The extracellular proteins obtained from the MSP- and MSP+ strain of $L$. pneumophila before and after passage through guinea pig lung were analyzed for proteolytic activity using a colorimetric assay with hide powder azure as the substrate.

* Number of times extracellular proteins of indicated strain were tested. 
from the macrophages intact. Some, possibly all, of the MSP may be processed intracellularly, and thus MSP may not be released by macrophages in a proteolytically active form. Along these lines, the finding that guinea pigs infected with $L$. pneumophila by aerosol develop a strong cell-mediated but not a strong humoral immune response to MSP, whereas guinea pigs infected subcutaneously with MSP develop strong cellmediated and humoral immune responses to MSP raises the possibility that only small linear determinants of MSP are presented to lymphocytes during infection with $L$. pneumophila. Such small linear determinants typically induce a cellular immune response that is often directed against unfolded linear epitopes, but not a humoral immune response, which is often directed against the tertiary structure of a protein antigen (13).

In this study, proteolytic activity of the MSP - mutant before and after guinea pig passage measured 0-1.9\% the level in the MSP+ strain; the levels were sufficiently low so as to be below the level of accurate detection by the colorimetric assay used. Independently, Szeto and Shuman have assayed residual protease activity in the MSP-strain described here and in five other independently derived MSP- strains and found that the proteolytic activity of all strains is $<0.2 \%$ that of the MSP+ strain (15a). Although present in small amounts, other proteases may yet contribute to the pathogenicity of $L$. pneumophila in Legionnaires' disease.

Our study raises the question as to the role of MSP, if any, in the biology of L. pneumophila. Humans appear to be accidental hosts for L. pneumophila, and since person-to-person transmission of Legionnaires' disease does not occur, humans are evidently not an important vector for dissemination of the bacterium. The primary reservoir for $L$. pneumophila in nature is the aquatic environment, where the organism is ubiquitous. As L. pneumophila has been shown to multiply in a variety of protozoa, aquatic unicellular organisms are presumably the bacterium's major hosts in nature. Possibly, MSP contributes to bacterial multiplication in protozoa or to extracellular survival in aquatic environments.

Although vaccination with MSP induces strong protective immunity in the guinea pig against lethal aerosol challenge with $L$. pneumophila, this protective immunogen is not required for the expression of virulence by L. pneumophila. Therefore, contrary to the traditional view, our findings demonstrate that a protective immune response need not necessarily be directed against a virulence determinant. Vaccination with MSP presumably enhances the capability of the immune system to detect and respond to L. pneumophila located intracellularly in mononuclear phagocytes. Preliminary studies in our laboratory have demonstrated that MSP is produced by $L$. pneumophila in infected human monocytes and that MSP epitopes are displayed on the monocyte surface (21). Immune lymphocytes might therefore detect intracellular $L$. pneumophila by recognizing MSP epitopes on the surface of infected mononuclear phagocytes. Such immune lymphocytes would then have the capability of selectively targetting and activating a cytotoxic antimicrobial response against infected host cells. By analogy, any molecule, even an "innocent bystander" molecule, that allows the host immune system to detect and act against pathogens sequestered intracellularly in mononuclear phagocytes may potentially serve as a protective immunogen against such pathogens.

\section{Acknowledgments}

We are grateful to Ms. Debora Gloria and Ms. Barbara Jane Dillon for expert technical assistance. We thank Dr. Daniel Clemens for providing affinity-purified monospecific anti-MSP antibody. We also thank Dr. Jeffrey Gornbein, UCLA Department of Biomathematics, for his expert statistical analysis of the data.

This work was supported by grant AI-22421 from the National Institutes of Health (NIH). Dr. Horwitz is Gordon MacDonald Scholar at the University of California, Los Angeles. During the time this work was performed, Dr. Blander was supported by NIH Training Grant AI-07323 and subsequently by a National Foundation for Infectious Diseases-Roerig Postdoctoral Fellowship in Nosocomial Infection Research.

\section{References}

1. Horwitz, M. A., and S. C. Silverstein. 1980. Legionnaires' disease bacterium (Legionella pneumophila) multiplies intracellularly in human monocytes. J. Clin. Invest. 66:441-450.

2. Wilkinson, H. W., A. L. Reingold, B. J. Brake, D. L. McGiboney, G. W. Gorman, and C. V. Broome. 1983. Reactivity of serum from patients with suspected legionellosis against 29 antigens of Legionellaceae and Legionella-like organisms by indirect immunofluorescence assay. J. Infect. Dis. 147:23-31.

3. Saha, A. K., J. N. Dowling, K. L. LaMarco, S. Das, A. T. Remaley, N. Olomu, M. T. Pope, and R. H. Glew. 1985. Properties of an acid phosphatase from Legionella micdadei which blocks superoxide anion production by human neutrophils. Arch. Biochem. Biophys. 243:150-160.

4. Saha, A. K., J. N. Dowling, A. W. Pasculle, and R. H. Glew. 1988. Legionella micdadei phosphatase catalyzes the hydrolysis of phosphatidylinositol 4,5-biphosphate in human neutrophils. Arch. Biochem. Biophys. 265:94-104.

5. Baine, W. B. 1988. A phospholipase C from the Dallas 1E strain of Legionella pneumophila Serogroup 5: purification and characterization of conditions for optimal activity with an artificial substrate. $J$. Gen. Microbiol. 134:489-498.

6. Cianciotto, N. P., B. I. Eisenstein, C. H. Mody, G. B. Toews, and N. C. Engleberg. 1989. A Legionella pneumophila gene encoding a species specific surface protein potentiates initiation of intracellular infection. Infect. Immun. 57:1255-1262.

7. Engleberg, N. C., C. Carter, D. R. Weber, N. P. Cianciotto, and B. I. Eisenstein. 1989. DNA sequence of mip, a Legionella pneumophila gene associated with macrophage infectivity. Infect. Immun. 57:1263-1270.

8. Muller, H. E. 1981. Enzymatic profile of Legionella pneumophila. J. Clin. Microbiol. 13:423-426.

9. Thorpe, T. C., and R. D. Miller, 1981. Extracellular enzymes of Legionella pneumophila. Infect. Immun. 33:632-635.

10. Conlan, J. W., A. Baskerville, and L. A. E. Ashworth. 1986. Separation of Legionella pneumophila proteases and purification of a protease which produces lesions like those of Legionnaires' disease in a guinea pig lung. J. Gen. Microbiol. 132:1565-1574.

11. Baskerville, A., J. W. Conlan, L. A. E. Ashworth, and A. B. Dowsett. 1986. Pulmonary damage caused by a protease from Legionella pneumophila. Br. J. Exp. Path. 67:527-536.

12. Dreyfus, L. A., and B. H. Iglewski. 1986. Purification and characterization of an extracellular protease of Legionella pneumophila. Infect. Immun. 51:736-743.

13. Blander, S. J., and M. A. Horwitz. 1989. Vaccination with the 
major secretory protein of Legionella pneumophila induces cell-mediated and protective immunity in a guinea pig model of Legionnaires' disease. J. Exp. Med. 169:691-705.

14. Keen, M. G., and P. S. Hoffman. 1989. Characterization of a Legionella pneumophila extracellular protease exhibiting hemolytic and cytotoxic activities. Infect. Immun. 57:732-738.

15. Quinn, F. D., and L. S. Tompkins. 1989. Analysis of a cloned sequence of Legionella pneumophila encoding a $38 \mathrm{kd}$ metallo-protease possessing haemolytic and cytotoxic activities. Mol. Microbiol. 3:797-805.

15a. Szeto, L., and H. A. Shuman. 1990. The Legionella pneumophila major secretory protein, a protease, is not required for intracellular growth or cell killing. Infect. Immun. 58:2585-2592.

16. Breiman, R. F., and M. A. Horwitz. 1987. Guinea pigs sublethally infected with aerosolized Legionella pneumophila develop humoral and cell-mediated immune responses and are protected against lethal aerosol challenge. A model for studying host defense against lung infections caused by intracellular pathogens. J. Exp. Med. 164:799811.
17. Laemmli, U. K. 1970. Cleavage of structural proteins during the assembly of the head of bacteriophage T4. Nature (Lond.). 227:680-685.

18. Towbin, H., T. Staehlin, and J. Gordon. 1979. Electrophoretic transfer of proteins from polyacrylamide gels to nitrocellulose sheets. Procedure and some applications. Proc. Natl. Acad. Sci. USA. 76:4350-4354.

19. Johnston, K. H., and J. B. Zabriskie. 1986. Purification and partial characterization of the nephritis strain-associated protein from Streptococcus pyogenes, group A. J. Exp. Med. 163:697-712.

20. Govindarajulu, Z. 1988. Statistical Techniques in Bioassay. Karger, Basel. 35-38.

21. Clemens, D. L., and M. A. Horwitz. 1990. Demonstration that Legionella pneumophila produces its major secretory protein in infected human monocytes and localization of the protein by immunocytochemistry and immunoelectron microscopy. Clin. Res. 38:480a. (Abstr.). 